

\title{
Cyanophora paradoxa mitochondrial tRNAs play a double game
}

\author{
Thalia Salinas-Giegé, Elodie Ubrig, Laurence Drouard
}

\section{To cite this version:}

Thalia Salinas-Giegé, Elodie Ubrig, Laurence Drouard. Cyanophora paradoxa mitochondrial tRNAs play a double game. Plant Journal, In press, 10.1111/tpj.15222 . hal-03175345

\section{HAL Id: hal-03175345 \\ https://hal.science/hal-03175345}

Submitted on 19 Mar 2021

HAL is a multi-disciplinary open access archive for the deposit and dissemination of scientific research documents, whether they are published or not. The documents may come from teaching and research institutions in France or abroad, or from public or private research centers.
L'archive ouverte pluridisciplinaire HAL, est destinée au dépôt et à la diffusion de documents scientifiques de niveau recherche, publiés ou non, émanant des établissements d'enseignement et de recherche français ou étrangers, des laboratoires publics ou privés. 
DR LAURENCE DROUARD (Orcid ID : 0000-0001-6537-524X)

Article type : Original Article

\title{
Cyanophora paradoxa mitochondrial tRNAs play a double game
}

\author{
Thalia Salinas-Giegé, Elodie Ubrig and Laurence Drouard1
}

Institut de biologie moléculaire des plantes-CNRS, Université de Strasbourg, Strasbourg, France

Running title : Mitochondrial RNA processing in glaucophytes

Keywords: mitochondrial RNA processing; organelles; evolution; green lineage

1To whom correspondence should be addressed. Email: Laurence.drouard@ibmpcnrs.unistra.fr - Postal address : Institut de biologie moléculaire des plantes, 12, rue du Général Zimmer, 67084 Strasbourg, France

\section{SUMMARY}

Present-day mitochondria derive from a single endosymbiosis of an $\alpha$-proteobacteria into a proto-eukaryotic cell. Since this monophyletic event, mitochondria have considerably evolved, and unique traits have been independently acquired in the different eukaryotic kingdoms. Mitochondrial genome expression and RNA metabolism have indeed highly diverged. Here, Cyanophora paradoxa, a freshwater alga considered a living fossil among photosynthetic organisms, represents an exciting model to study mitochondrial

This article has been accepted for publication and undergone full peer review but has not been through the copyediting, typesetting, pagination and proofreading process, which may lead to differences between this version and the Version of Record. Please cite this article as doi:

\subsection{1/TPJ.15222}

This article is protected by copyright. All rights reserved 
gene expression evolution. As expected, fully mature tRNAs are released from primary transcripts to function in mitochondrial translation. Besides, we show that these tRNAs also take part in an mRNA processing punctuation mechanism in a nonconventional manner leading to mRNA-tRNA hybrids with a CCA triplet at their 3'-extremities. In this case, tRNAs are likely used as stabilizing structures impeding mRNA degradation by exonucleases. From our data, we propose that the present-day tRNA-like elements (telements) found at the 3 '-termini of mitochondrial mRNAs in land plants originate from true tRNAs as those observed in the mitochondria of this basal photosynthetic glaucophyte.

\section{INTRODUCTION}

The mitochondrion is central to eukaryotic bioenergetics, and studying mitochondrial genome expression and its evolution is fundamental. It is generally admitted that all mitochondria originated from a single $\alpha$-proteobacterium engulfed in a proto-eukaryotic cell and evolved as a subcellular organelle (Roger et al., 2017). While most of the genes of the ancestral endosymbiotic bacteria have been either lost or transferred to the nuclear genome, a genome relic of the ancestral bacterial DNA still exists in the mitochondrion and encodes a small number of essential protein genes. Despite their monophyletic origin, mitochondrial genomes developed independently in evolutionarily divergent organisms. This evolution leads to a fascinating complexity of mitochondrial DNA size, organization, and expression (e.g. (Smith and Keeling, 2015), (Pearce et al., 2017),(Hammani and Giegé, 2014)) that we still need to unravel.

Organisms belonging to the Archaeplastida (also named Plantae) illustrate very well this vast diversity. This group comprises land plants, chlorophytes, rhodophytes, and glaucophytes. Indeed, their mitochondrial DNA exhibits a wide range of sizes, from very large as in the land plant Silene conica (circa $11 \mathrm{Mb}$ ), large as in a vast majority of other seed plants (e.g., Arabidopsis thaliana, 367 kb or Zea mays, 536-740 kb) (Sloan et al., 2012), to significantly reduced ones as in the red alga Chondrus crispus (25.8 kb) (Leblanc et al., 1995) or the green alga Chlamydomonas reinhardtii (15.8 kb) (Grant and 
Chiang, 1980). This size variation also correlates with other disparities. Large mitochondrial genomes usually possess large intergenic sequences, contain group II introns and, are transcribed from multiple promoters throughout the genome. Moreover, a cascade of post-transcriptional events is necessary to produce mature transcripts, often endonucleolytically processed at their 3 ' extremities, where stem-loop structures stabilize them (Hammani and Giegé, 2014). By contrast, for instance, the small genome of $C$. reinhardtii is very compact, with very short intergenic sequences and no introns. It is transcribed from two promoter regions, intercistronic maturation generates adjacent 5'and 3'-termini, all mRNAs have no 5'-UTR, and polyC-rich added tails at their 3'extremities (Salinas-Giegé et al., 2017).

Cyanophora paradoxa represents another prime model organism. Belonging to the glaucophytes, the most basal group of Archaeplastida, this freshwater alga is considered a living fossil among photosynthetic organisms. It still contains two plastids with unique cyanobacterial characters, such as the remnants of a peptidoglycan cell wall, carboxysome-like bodies, and phycobilisome antennas (Jackson et al., 2015). The analysis of its nuclear genome allowed to elucidate the origin of photosynthesis (Price et al., 2012). Its $51.6 \mathrm{~Kb}$ mitochondrial genome (Price et al., 2012) is 74\% AT-rich and encodes 38 protein-coding genes, 10 unidentified Open Reading Frames (ORFs), 3 ribosomal RNA (rRNA) genes, and 26 distinct tRNA genes (Figure S1). These tRNA genes a priori may correspond to a full set to allow the reading of all codons according to the universal genetic code, with the notable exception of the missing tRNA ${ }^{T h r}$ genes. In compliance with the orientation of transcription of each gene, we can postulate that the $C$. paradoxa mitochondrial genome is transcribed as two large polycistronic RNAs and two shorter ones. As the tRNA genes are interspersed throughout the circular genome (Figure S1), we wondered whether their processing could be used to release several mature mRNAs as is often the case in the mitochondrial metazoan world, a process called the tRNA punctuation model (Ojala et al., 1980), (Anderson et al., 1981), (Ojala et al., 1981).

Here, we analyzed the processing of $C$. paradoxa mitochondrial mRNAs and tRNAs using, in particular, 3'-RACE (Rapid Amplification of cDNA Ends) and cRT-PCR (circular Reverse Transcription-Polymerase Chain Reaction) methods. Our results shed light on a singular characteristic of many mRNA transcripts. When a tRNA gene follows an mRNA 
coding sequence, the mRNA's $3^{\prime}$ extremity is not released from the primary transcript by the tRNA 5'-end processing. Instead, the 3 '-end tRNA processing occurs, and a CCA triplet is added post-transcriptionally, likely by a tRNA nucleotidyltransferase. Consequently, the mRNAs' 3 ' extremities harbor highly folded tRNA structures likely to stabilize them. This processing strategy acts in synergy with the same tRNAs' classical processing to release mature functional tRNA species used in protein synthesis. In the latter case, mRNAs are not anymore protected against exonucleases and are likely rapidly degraded. Thus, our analyses strongly suggest that $C$. paradoxa mitochondrial tRNAs participate both in protein synthesis, their primary role, and in the stabilization of mature mRNAs. Finally, we also report in this work the existence of polycistronic mRNAs encoding several proteins, raising the question of the translation initiation/reinititation mechanisms in C. paradoxa mitochondria. Taken together, our findings provide new insights into the multiple ways the evolution has developed to express essential mitochondria-encoded genes.

\section{RESULTS}

\section{C. paradoxa mitochondrial tRNA genes are dispersed throughout the genome}

Generally, in Archaeplastida, mitochondrial tRNA genes are often in clusters as illustrated for the land plant $A$. thaliana (Unseld et al., 1997), the bryophyte Marchantia polymorpha (Ohyama, 1996), or the rhodophyte C. crispus (Leblanc et al., 1995), (Figure S2). Also, their number is variable and can be highly reduced. For instance, only three tRNA genes are present on the vast mitochondrial genome of the land plant Silene noctiflora (Wu et al., 2015) or the very compact genome of the green alga C. reinhardtii (Grant and Chiang, 1980). Here, first, in the C. paradoxa mitochondrial genome, with 30 tRNA genes corresponding to 26 tRNA isoacceptors, the set is almost complete, and a priori only tRNA ${ }^{\text {Thr }}$ gene(s) are lacking. Otherwise, on the genome's circular map (Figure S1), we corrected a tRNAlle gene with a CAT anticodon wrongly annotated as a tRNA ${ }^{\text {Met }}$ gene in the original work of Price et al. (Price et al., 2012). This correction is also accessible in (Cognat et al., 2013). Second, except for four adjacent tRNA genes, the other ones are either alone or, in a few cases, close to a second tRNA gene. Indeed, most tRNA genes are spread along the relatively compact genome (Figure S1), and 18 tRNA genes are located downstream of mRNAs in their very close vicinity. The average distance between 
the stop codon of the mRNA's coding sequence and the 5 '-end of the tRNA is $25 \mathrm{nt}$, except tRNA ${ }^{\text {Ser1 }}$ located 328 nt downstream of orf229 (Figure 1). This organization is a peculiar feature in the green lineage world. It is reminiscent of the situation observed in the human mitochondrial genome where most mRNAs are immediately adjacent at their $3^{\prime}$ end to tRNA genes. In this case, processing of primary transcripts by the endoribonucleases RNase P and RNase $Z$ at the level of the 5'- and 3'-extremities of tRNAs allows, in most cases, to release not only mature tRNAs but also mature mRNAs and rRNAs (Ojala et al., 1980), (Anderson et al., 1981), (Ojala et al., 1981). Therefore, we wondered whether tRNA processing is essential in $C$. paradoxa mitochondria to release mature mRNAs.

\section{The 3'-extremity of several C. paradoxa mitochondrial mRNAs lies at the 3'- mature end of the adjacent tRNA}

We first carried out 3'-RACE experiments on a few mRNA candidates to explore the potential role of tRNAs processing in the release of mature mRNAs (Figure S3A). As C. paradoxa mitochondrial DNA presents a high A/T content of around $75 \%$, we focused our analysis on mRNAs where the possibility to design specific oligonucleotides was possible. The first mRNA analyzed codes for the ribosomal protein rps4. The UAA stop codon of the rps 4 coding sequence is $10 \mathrm{nt}$ upstream of the tRNA ${ }^{\text {Tyr }}$ gene (Figure 1). The 3'-RACE experiment shows that, in most cases, the 3'-UTR of rps4 mRNA comprises the short intergenic sequence of $10 \mathrm{nt}$ plus the full-length tRNATyr to which a CCA sequence has been post-transcriptionally added (Figure 2A). Out of 47 clones (Figure 2B), 25 show the same 3'- termini, 13 sequences present a truncated CCA triplet, and 7 are shortened by up to $3 \mathrm{nt}$ before the CCA end. Notably, the 3'-extremity of the RNAs stops neither at the 5 '-extremity of the tRNA molecule nor at the 3 '-end of the coding sequence (i.e., only one sequence ends at the 3 '-extremity of rps4 coding sequence but with a truncated stop codon). By contrast, based on 3'-RACE clones, we found around half of the 5S rRNA 3'extremities ending within the last $6 \mathrm{nt}$ of the predicted 3'-termini (Figure 2C). The remaining sequences correspond to truncated versions of $5 S$ rRNA, and none of them ends at the $3^{\prime}$ - extremity of the adjacent tRNA ${ }^{\text {Leu1 }}$ as observed in the case of rps4. Conversely, the 5'-RACE experiment using an oligonucleotide specific to tRNALeu1 confirmed the precise endonucleolytic cleavage by RNase $P$ to release the 5 '-end of the 
tRNA molecule. We performed a similar analysis for 5 other mRNAs to confirm the peculiar characteristic found for rps4 mRNA, i.e., the existence of a 3'-UTR comprising a full-length tRNA. These mRNAs (rps10, nad4, nad5, nad6, cox2) are all followed by an adjacent tRNA (Figure 2D). Previously published data for nad4 were also considered (Salinas-Giegé et al., 2017). As for rps4, 66 to $80 \%$ of the 3'-RACE clones possess tRNA sequences downstream of the mRNA sequences, and, on average, $60 \%$ of them present a post-transcriptionally added CCA triplet at their extremities. Conversely, we did not detect sequences ending either at the stop codons level or the 5'-extremity of tRNA molecules. The 3'-RACE methodology relies on the ligation of a primer to the 3'-end of an RNA, a step that could lead to artifacts. We thus used a second approach, circular RTPCR (cRT-PCR), to validate the results (Figure S3B). As determined for nad4, nad5, and nad6 mRNA coding sequences are followed by full-length tRNAs ending with CCA triplet, thus corroborating the 3'-RACE data (Figure S4 and Figure S5).

Nevertheless, as both approaches (3'-RACE and cRT-PCR) relies on compatible 3' phosphate extremities for ligation, we cannot entirely exclude that, due to unexpected pathways and/or nucleases, the mRNAs' 3'-ends are not compatible with the ligation step and thus, cannot be observed. A northern blot performed with a probe specific to nad4 mRNA shows a single band with a size of circa 1540 nt (Figure 2E, Figure S8). If we hypothesize a 5'-UTR of 25 nt (Figure S4), the length of the hybrid nad4 mRNA-tRNALeu3 is circa $1580 \mathrm{nt}$, that of nad4 mRNA $1500 \mathrm{nt}$ (if we postulate a 3' end at the 5' tRNA extremity level). The low resolution on the northern blot does not allow us to differentiate between the two possibilities. However, as we already demonstrated the existence of the nad4 mRNA-tRNALeu3 hybrid, the presence of a single band argues in favor of the existence of the nad4-tRNALeu3 hybrid isoform only.

Finally, this allowed us to show that at least for these 4 mRNAs (Figure S4), the 5'-UTRs of most clones are in the majority relatively short (average length of $25 \mathrm{nt}$, Figure $2 \mathrm{~F}$ ) and $\mathrm{A} / \mathrm{T}$ rich $(>85 \%)$.

Altogether, these analyses strongly suggest that in $C$. paradoxa mitochondria, tRNAs punctuate RNAs processing but not in the same way as in metazoan mitochondria. Instead of releasing the 3'-extremity of a mature mRNA by 5'-end tRNA processing, the cleavage occurs at the 3 '-end of the tRNA to maintain a strong folded structure at the 3 'end of the mRNA. 


\section{Polycistronic and individual mRNAs coexist in C. paradoxa mitochondria}

As described above, many tRNA genes follow protein-coding genes in C. paradoxa mitochondrial DNA. Nevertheless, this is not always the case (Figure S1), (Price et al., 2012). At least two other situations exist.

First, a subset of mRNAs is not followed by tRNAs but rather by inverted repeat structures. This is, for instance, the case for cox1 and nad7 mRNAs (Figure 3). Reanalyzing 3' RACE data that we previously published (Salinas-Giegé et al., 2017), the majority of 3'-termini of cox1 mRNA were located at the precise $3^{\prime}$ extremity of a stemloop structure or in its vicinity (Figure 3). The 3 ' termini of nad7 mRNA were less precisely defined, with $29 \%$ of them located between the stop codon of nad7 CDS and the 5 -extremity of an inverted repeat structure: $14 \%$ within the stem-loop, and $46 \%$ of the sequences ending at or near to the 3'- end of the inverted repeat sequence, (Figure 3). A few clones corresponding to truncated nad7 sequences were also found. We already found such a situation in higher plant mitochondria, where stem-loop structures are used as processing sites and stabilizing signals (Hammani and Giegé, 2014), (Dombrowski et al., 1997). As a whole, these results show that classical processing at the level of stemloop structures can also occur in C. paradoxa to provide mitochondria with stable mRNAs. Second, some coding sequences follow each other with very few nucleotides between them (i.e., between the stop codon and the initiation codon of two successive coding sequences), or even they overlap (Figure $4 \mathrm{~A}$ ). It is presently unknown how the $C$. paradoxa mitochondrial deals with this situation. Two major hypotheses can be envisaged: i) precursor transcripts are precisely cleaved between each coding sequence before translation occurs (implying in some cases the production of truncated mRNAs missing the initiation or the stop codon), or ii) long polycistronic mRNAs remain uncleaved further requiring for instance either ribosome scanning or peculiar internal ribosome entry sites to produce each protein independently. As a first step towards understanding the process, we determined by CRT-PCR whether individual mRNAs and/or large polycistronic mRNAs exist in the region encompassing nad1-rps13-rps11rpl5-orf99-rpl6-tRNAMete (Figure 4A). When we used a pair of primers allowing the potential amplification of the extremities of nad1 (P1+P2) or rpl6 (P3+P4), only nonspecific products were obtained (Figure 4B). By contrast, with the pair of primers (P1+P4), 
we got a PCR product corresponding to the 5 '-extremity of nad1 fused to the 3 '-end of rpl6, including a mature tRNAMete with a CCA triplet (Figures. 4B and $4 C$ ). This result strongly suggests the absence of internal cleavage in the long RNA starting from the 5'UTR of nad1 (of around $40 \mathrm{nt}$, Figure 2E) to the $3^{\prime}$ extremity of tRNAMete and consequently the existence of a long polycistronic mRNA. To validate this result, we analyzed data previously published by Price et al. (Price et al., 2012), and mapped total Illumina RNA-seq on the C. paradoxa mitochondrial DNA. In agreement with the CRTPCR results, the mapping of the reads of the nad1-rpl6 region shows no individually processed mRNAs but rather a large transcript covering the whole area (Figure 4D). As a counter-example, the processing site present between the 3 '-end of the small subunit rRNA and the 5 '-end of orf535 is easily identifiable, thus validating the approach. It is to note that the full mitochondrial genome coverage appeared heterogeneous likely due to strong tRNA folding. Generally, we observed a low coverage for tRNAs and 3'-extremities of RNAs. Nevertheless, we could identify polycistronic mRNAs in other regions such as sdh3-rps12-rps7-rps10, nad3-orf162, or rpl2-rps19-rps3-rpl16 (Figure S6). By contrast, a cleavage site seems to exist between orf162 and sdh4, two RNAs separated by $161 \mathrm{nt}$.

Taken together, these analyses strongly suggest that both individual and polycistronic mRNAs can be generated and coexist in C. paradoxa mitochondria.

\section{Mature tRNAs are expressed from $C$. cyanophora mitochondrial tRNA genes}

Except for the lacking tRNA ${ }^{\text {Thr }}$ gene(s), the set of $C$. paradoxa mitochondrial tRNA genes seems a priori sufficient to ensure mitochondrial translation. In photosynthetic organisms, the import of cytosolic tRNAs to compensate for the lack of mitochondrially-encoded tRNAs is an overall process (Huot et al., 2014). Here, the import of tRNA ${ }^{\text {Thr }}$ cytosolic counterparts is likely to occur. Besides that, as described above, we found tRNA precursors processed at their 3 '-extremity without a cleavage at their 5'-end likely to stabilize mRNA. Although we cannot exclude the import of other cytosolic tRNA species to replace these tRNAs, another simple hypothesis is that the proportion of tRNAs linked to mRNAs is very low and corresponds to the amount of translated mRNAs while most tRNA precursors are correctly processed at both 5'- and 3'-termini to produce mature and functional tRNAs. To validate this hypothesis, we determined tRNA extremities by cRTPCR analysis (Figure 5A). After the circularization of total C. paradoxa RNAs, specific 
products were amplified by RT-PCR (Figure 4B) using pairs of primers specific to tRNA ${ }^{\text {Leu3 }}$, tRNA ${ }^{A s n}$, tRNA ${ }^{\text {Tyr }}$, and tRNAGly (Table S1). The three first are located downstream mRNAs and the last one downstream tRNA Asn. In all cases, after cloning and sequencing, the deduced sequences show the existence of faithfully processed mature tRNAs. The presence of correct 5'- and 3'-extremities and a CCA triplet at the 3'end attest to this (Figure $5 \mathrm{~B}$ and Figure S7). This result shows that classical tRNA processing by RNase $P$ first and then RNase $Z$ does occur to generate a sufficient amount of mature tRNAs to have an optimal translation process. If this is true, we can hypothesize we will detect much more mature tRNA than the corresponding mRNA-tRNA. In agreement with this, when we performed northern blots on C. cyanophora total RNAs with probes specific to tRNA ${ }^{\text {Leu3 }}$ or tRNAAsn, we did not obtain signals at a size corresponding to nad4-tRNA $A^{\text {Leu3 }}$ (1552 nt) or nad5-tRNAAsn $(2113 \mathrm{nt})$, respectively. Still, we observe strong signals at the level of tRNA ${ }^{\text {Leu3 }}$ or tRNA Asn (Figure $5 \mathrm{C}$ ). This shows that the relative amount of a mature tRNA as compared to its mRNA-tRNA counterpart is huge. This contrast is expected to avoid tRNA shortage and to allow optimal mt translation.

\section{DISCUSSION}

While the number of fully sequenced mitochondrial genomes increases every day, the question of how mitochondrial gene expression has evolved since the monophyletic $\alpha$ proteobacterial endosymbiotic event remains. Here, we focused our work on the mitochondrial genome expression of the most basal member of Archaeplastida, the glaucophyte $C$. paradoxa. It represents the closest living organism of the cyanobacterial endosymbiosis leading to the primary plastid common to the Archaeplastida (i.e., glaucophytes, red and green algae, and land plants).

With a size of about $51 \mathrm{kbp}$, the mitochondrial genome of C. paradoxa (Price et al., 2012) is relatively compact with a high gene density, a characteristic shared with rhodophyte and chlorophyte mitochondrial genomes. Otherwise, contrary to these genomes, the $C$. cyanophora mitochondrial DNA organization resembles more the generally highly compact mitochondrial genome of animals where most tRNA genes are interspersed between protein-coding genes and located 1-2 nt downstream of protein-coding sequences (Lavrov and Pett, 2016). In this latter case, tRNAs are used as punctuation to 
release rRNAs and mRNAs (Ojala et al., 1980), (Anderson et al., 1981), (Ojala et al., 1981). This occurs via endonucleolytic cleavages of the three human polycistronic primary transcripts by RNase P and RNase Z (named ELAC2 in human) at the 5'- and 3'extremities of tRNA, respectively. Further processing steps, such as polyadenylation, are then required to generate mature, functional, and stable mRNAs. No experimental data on the transcription initiation sites and transcription process exist in C. paradoxa mitochondria. Still, according to its genome organization, it looks likely that transcription starts at a limited number of promoters and generates long primary polycistronic transcripts encompassing large portions of the genome (Figure S1). In C. paradoxa, we report that although punctuating the mitochondrial genome, tRNA genes are not used for the same purpose.

Indeed, in our model (Figure 6), classical tRNA processing carried out by RNase $\mathrm{P}$ and RNase $Z$ does occur and represents the main processing pathway to generate a sufficient amount of mature tRNAs for mitochondrial translation. Besides this, a tiny proportion of precursor transcripts is first cleaved by RNase $Z$ at the $3^{\prime}$-end of the folded tRNA sequence, and a tRNA nucleotidyltransferase adds a CCA triplet. In this latter case, precursor tRNAs are likely less or not accessible to RNase P. Our results are consistent with previous analysis on land plants mitochondria. Fully processed tRNAs result from the 5 '-end cleavage achieved by RNase $P$ primarily preceding the 3 '-end processing by RNase Z (Mörl and Marchfelder, 2001), (Sieber et al., 2011). However, to a lesser extent, the cleavage by RNase $Z$ and the CCA addition by the tRNA nucleotidyltransferase can precede the cleavage by RNase $\mathrm{P}$ (Sieber et al., 2011). Also, in vitro cleavage assay of a precursor tRNA performed with a recombinant Arabidopsis mitochondrial RNase $P$ is inhibited by the presence of a CCA triplet at the 3'- extremity of the tRNA precursor molecule (Gobert et al., 2013). In land plants, the mitochondrial RNase P is not a ribonucleoprotein complex with a catalytic RNA but rather a PROteinaceous RNase $P$ called PRORP (Gobert et al., 2010), as in human mitochondria. A candidate RNase P with a PRORP catalytic domain has been identified in C. paradoxa. Still, its mitochondrial location, as well as the proof of its activity, remains to be established (Lechner et al., 2015). Our data suggest that this enzyme's activity is likely affected by the addition of a CCA at the 3'- extremity of the tRNA precursor. Still, definitive evidence will await for its detailed characterization. Additionally, it is worth mentioning that several post- 
transcriptional modifications are or can be added to tRNA precursor molecules (e.g., Hopper, 2013; Torres et al., 2015). Therefore, we can wonder whether, in C. paradoxa, some of the post-transcriptional modifications likely present on mature mitochondrial tRNAs can be added already to the tRNA structures still linked to mRNAs and if their presence can represent another way to slow down tRNA processing.

Two mitochondrial RNases Z involved in tRNA 3'-end processing have been identified in Arabidopsis (Canino et al., 2009). Based on sequence homology, only one potential candidate protein with a predicted tRNase $\mathrm{Z}$ motif could be retrieved from $C$. paradoxa nuclear genome (g18909.t1, Protein ID: 19276), its location within the cell is currently unknown. Further studies are required to understand if the mitochondrial RNase $Z$ of glaucophytes can cleave precursor tRNA molecules unprocessed at their 5'-extremities. In agreement with this, numerous tRNA-like secondary structures called t-elements are located close to RNAs extremities (Hanic-Joyce et al., 1990), (Bellaoui et al., 1997) and are substrates of RNase Z in higher plant mitochondria (Canino et al., 2009). Indeed, in Arabidopsis mitochondria, the primary role played by t-elements in the formation of mRNA termini upon processing by RNase $P$ and RNase $Z$ has been determined, and their involvement in mRNA stability has also been proposed (Forner et al., 2007). Several t-elements potentially involved in RNA processing and/or RNA stability have been recently identified on the potato mitochondrial genome (Varre et al., 2019). For instance, a chloroplast-like tRNACys gene located within the 3'-termini of the CcmC coding sequence seems to act as an RNA processing signal. The corresponding mature and stable tRNA, a priori not present in mitochondria, is likely dispensable for mitochondrial translation. In Arabidopsis, this tRNA gene, located at the same position, has become a pseudogene and acts as a t-element for RNA processing (Forner et al., 2007). These data raise questions about the origin of t-elements and their role as processing signals and/or stabilizing factors. Considering our present knowledge on mitochondrial genome expression and the data presented in this study on C. paradoxa, it is tempting to speculate that, beyond their role in translation, the location of tRNA genes evolved to punctuate RNA processing in the highly compacted genome such as in animals but also to serve as stabilizing factors thanks to their highly folded structure in others. This latter role, mainly developed in Archaeplastida, is attested here in the basal member $C$. cyanophora, where many tRNAs still play a double role. Recombination events, prone to 
be frequent in higher plant mitochondrial genomes, caused either duplication or disappearance of tRNA genes during evolution. It appears that the import of nuclearencoded tRNA species compensated for this loss of tRNA genes. Such an event may have accelerated the mutation rate in a subset of tRNA genes that became tRNA pseudogenes and progressively in what we now call t-elements, elements still involved in RNA processing and stabilization.

The presence of mRNA-tRNA molecules in C. paradoxa mitochondria also reminds us of the situation occurring in positive-strand RNA viruses, which possess at their 3'-end a tRNA-like structure (called TLS). The first to be identified is the turnip yellow mosaic virus that contains a TLS highly resembling a canonical tRNA (Yot et al., 1970). A large variety of functions have been attributed to TLS in the course of viral infection. These functions include the enhancement of translation and stability but also aminoacylation properties (Dreher, 2009). As most C. paradoxa mitochondrial mRNA-tRNA hybrids possess a CCA triplet at their 3'- extremity, another open question is to know if such molecules are charged in vivo in the organelles and used in the translation process. Mature tRNAs are a priori sufficient to fulfill the translation process, but we cannot exclude the presence of aminoacylated mRNA-tRNA hybrids.

Finally, the observation that endonucleolytic cleavage does not release monocistronic mRNAs from some polycistronic mRNAs is intriguing. It also questions how the translation process occurs to produce multiple proteins from a polycistronic mRNA. Intergenic regions between the coding sequences are minimal (and even overlaps) and exclude the presence of Internal Ribosome Entry Sites (IRES) commonly used in RNA viruses and also found in eukaryotic mRNAs (Hellen and Sarnow, 2001). Leaky scanning or initiation at alternative selected translation start codons (Ryabova et al., 2006) (Hinnebusch, 2011) is another possibility. The intergenic regions between near-located coding sequences are very $\mathrm{A} / \mathrm{T}$ rich. However, we could not determine a motif on the $C$. paradoxa mitochondrial genome involved in translation initiation or supporting an alternative translation hypothesis at particular AUG start codons. Future research aiming at deciphering the translation initiation process occurring in this model organism thus appears essential to our understanding of biological systems.

\section{EXPERIMENTAL PROCEDURES}




\section{Glaucophyta strain and RNA extraction}

The Scandinavian Culture Collection of Algae and Protozoa (SCCAP) provided us with the Cyanophora paradoxa (K-0262) strain. RNA was prepared from whole cells using TRI Reagent ${ }^{\circledR}$ (Molecular Research Center) according to the manufacturer's instructions.

\section{RNA and bioinformatics analyses}

The 5' and 3' RACE and CRT-PCR analyses were performed using Sanger sequencing (Salinas-Giegé et al., 2017). Briefly, for 3' RACE, 4 to $6 \mu \mathrm{g}$ of total RNA were ligated at the 3'-end to an adaptor and cDNA was synthesized with SuperScript IV (Invitrogen) primed by the complementary adaptor RT primer. PCR was performed with gene-specific primers and the RT primer. For 5' RACE, the first-strand CDNA synthesized above was C-tailed with the terminal deoxynucleotidyl-transferase (Invitrogen) in the presence of dCTP. PCR was performed with a gene-specific forward primer and oligo(dG). For cRTPCR, 4 to $6 \mu \mathrm{g}$ of total RNA was circularized by T4 RNA ligase (New England Biolabs) prior to reverse transcription and PCR using gene-specific oligonucleotides. All PCR products were cloned into pGEM-T® Easy vector (Promega) and sequenced using Sanger technology.

Sequence alignments were generated with MacVector software (https://macvector.com) as previously described (Salinas-Giegé et al., 2017). The RNAfold program (http://rna.tbi.univie.ac.at/) was used to identify the putative secondary structure. RNASeq reads retrieved from SRA (SRR363339) were mapped on the C. paradoxa mitochondrial genome (NC_017836) using the Geneious bioinformatics software.

\section{Northern blot}

For tRNA detection by northern blots, $10 \mu \mathrm{g}$ of total RNAs were separated on denaturing formaldehyde-agarose gel, transferred onto Hybond $\mathrm{N}+$ membrane as recommended by the manufacturer (GE Healthcare Life Sciences) and hybridized to ${ }^{32} \mathrm{P}$ radiolabelled oligonucleotide probes in $6 \mathrm{xSSC}, 0.5 \%$ SDS at $45^{\circ} \mathrm{C}$. Washing conditions were: 2 times $10 \mathrm{~min}$ in $2 \times \mathrm{SSC}$ and 1 time $30 \mathrm{~min}$ in $2 \times \mathrm{SSC}, 0.1 \%$ SDS at the hybridization temperature.

For nad4 mRNA detection, the same blot was hybridized with a nad4 specific probe (DIG Easy Hyb kit, Roche) according to the manufacturer's instructions. The probe is a PCR 
product that is digoxigenin labelled using PCR DIG Probe Synthesis kit (see Table S1 for the primers).

\section{Miscellaneous}

The oligonucleotide sequences used in this study are listed in Supplementary Table S1. They were synthesized by the company Integrated DNA Technologies IDT.

\section{DATA AVAILABILITY STATEMENT}

All the relevant data can be found within the manuscript and its supporting materials.

\section{ACKNOWLEDGMENTS}

This work benefits from funding from the state managed by the French National Research Agency as part of the Investments for the future program (MITOCROSS ANR11-LABX-0057). It was also supported by the Centre National de la Recherche Scientifique (CNRS) in association with the University of Strasbourg. We wish to thank the Gene Expression and the bioinformatic platforms of IBMP for technical support.

\section{AUTHOR CONTRIBUTIONS}

TS performed 3' RACE experiments, designed work, analyzed the sequencing data, critically participated in the writing of the manuscript. EU performed $5^{\prime}$ and $3^{\prime}$ RACE experiments, cRT-PCR experiments, cloning and northern blots. LD supervised the work, design the experiments, analyzed the data, wrote the manuscript.

\section{CONFLICT OF INTEREST STATEMENT}

No potential conflict of interest was reported by the authors.

\section{SUPPORTING INFORMATION}

Uncropped blots and gels are provided in Supplementary Figure S8. Alignments of 3' RACE and CRT-PCR sequences are provided in Supplementary Figure S9.

Additional Supporting information may be found in the online version of this article. 
Figure S1. Schematic map of the circular mitochondrial genome of $C$. paradoxa. The map is adapted from (Price et al., 2012). Protein-coding genes and unidentified ORFs are depicted with blue arrows, rRNA genes with thin green arrows and tRNA genes with thin red arrows. When several isoacceptor tRNA genes exist, they are numbered. The correct annotation of the tRNAlle gene with a CAT anticodon is mentioned. The elongator and initiator tRNAMet genes are $\mathrm{Me}$ and $\mathrm{Mi}$, respectively.

Figure S2. Schematic map of mitochondrial genomes from Chondrus crispus (Z47547), Marchantia polymorpha (M68929) and Arabidopsis thaliana (Y08501).

Figure S3. Ethidium bromide-stained gels of the PCR products obtained (A) by 3' RACE and (B) CRT-PCR. M: DNA ladder.

Figure S4. Mapping of the mRNA extremities by CRT-PCR. (A), Scheme of the CRT-PCR strategy. First, RNAs are ligated using T4 RNA ligase. Second, cDNA is synthesized thanks to primer $\mathrm{P} 1$ and $\mathrm{PCR}$ products are obtained after PCR amplification with primers P1 and P2. (B), Sample sequences of clones were obtained from the cRT-PCR amplification products of nad4, nad5 and nad6 mRNAs. The 3'- and 5'- extremities of the RNA molecule are presented and the added CCA triplet to tRNA molecules is framed.

Figure S5. Screenshots of the alignment of 5' and $3^{\prime}$ extremities of nad4 (A), nad5 (B) and nad6 $(C)$, as deduced from cRT-PCR sequences.

Figure S6. Screenshots of the alignment of small RNA-seq data retrieved from SRA (SRR363339) on (A) srrn-orf535, (B) sdh3-rps12-rps7-rps10, (C) nad3-orf164-sdh4 and, (D) rpl2-rps19-rps3-rpl16 regions of $C$. paradoxa mitochondrial genome, using Geneious genome browser.

Figure S7. Mapping of tRNA extremities by CRT-PCR. Sample sequence of one of the clones obtained from cRT-PCR amplification products for tRNALeu3, tRNAAsn, tRNAGly, and tRNA ${ }^{\text {Tyr. }}$. The 3'- and 5'- extremities of tRNAs are indicated. The post-transcriptionally added CCA triplet is framed.

Figure S8. Raw data of blots and gels presented in this work.

Figure S9. Screenshots of (A) the 3' RACE and (B) the cRT-PCR sequence alignments with MacVector software. The top row corresponds to the sequence of reference. The left column indicates the clone's names. The black sequence corresponds to what is aligned to the reference, and the gray sequence corresponds to what no longer aligns.

Table S1. Primers used in this work. 


\section{REFERENCES}

Anderson, S., Bankier, A.T., Barrell, B.G., de Bruijn, M.H., Coulson, A.R., Drouin, J., Eperon, I.C., Nierlich, D.P., Roe, B.A., Sanger, F., Schreier, P.H., Smith, A.J., Staden, R. and Young, I.G. (1981) Sequence and organization of the human mitochondrial genome. Nature, 290, 457-465.

Bellaoui, M., Pelletier, G. and Budar, F. (1997) The steady-state level of mRNA from the Ogura cytoplasmic male sterility locus in Brassica cybrids is determined posttranscriptionally by its $3^{\prime}$ region. EMBO J, 16, 5057-5068.

Canino, G., Bocian, E., Barbezier, N., Echeverria, M., Forner, J., Binder, S. and Marchfelder, A. (2009) Arabidopsis encodes four tRNase Z enzymes. Plant Phys, 150, 1494-1502.

Cognat, V., Pawlak, G., Duchêne, A.M., Daujat, M., Gigant, A., Salinas, T., Michaud, M., Gutmann, B., Giegé, P., Gobert, A. and Maréchal-Drouard, L. (2013) PlantRNA, a database for tRNAs of photosynthetic eukaryotes. Nucleic Acids Res, 41, D273-279.

Dombrowski, S., Brennicke, A. and Binder, S. (1997) 3'-inverted repeats in plant mitochondrial mRNAs are processing signals rather than transcription terminator. EMBO J., 16, 5069-5076.

Dreher, T.W. (2009) Role of tRNA-like structures in controlling plant virus replication. Virus Res, 139, 217-229.

Forner, J., Weber, B., Thuss, S., Wildum, S. and Binder, S. (2007) Mapping of mitochondrial mRNA termini in Arabidopsis thaliana: t-elements contribute to $5^{\prime}$ and 3' end formation. Nucleic Acids Res, 35, 3676-3692.

Gobert, A., Gutmann, B., Taschner, A., Gossringer, M., Holzmann, J., Hartmann, R.K., Rossmanith, W. and Giegé, P. (2010) A single Arabidopsis organellar protein has RNase P activity. Nature Struc Mol Biol, 17, 740-744.

Gobert, A., Pinker, F., Fuchsbauer, O., Gutmann, B., Boutin, R., Roblin, P., Sauter, C. and Giegé, P. (2013) Structural insights into protein-only RNase P complexed with tRNA. Nature Comm, 4, 1353. 
Grant, D. and Chiang, K.S. (1980) Physical mapping and characterization of Chlamydomonas mitochondrial DNA molecules: their unique ends, sequence homogeneity, and conservation. Plasmid, 4, 82-96.

Hammani, K. and Giegé, P. (2014) RNA metabolism in plant mitochondria. Trends Plant Sci, 19, 380-389.

Hanic-Joyce, P.J., Spencer, D.F. and Gray, M.W. (1990) In vitro processing of transcripts containing novel tRNA-like sequences ('t-elements') encoded by wheat mitochondrial DNA. Plant Mol Biol, 15, 551-559.

Hellen, C.U. and Sarnow, P. (2001) Internal ribosome entry sites in eukaryotic mRNA molecules. Genes Dev, 15, 1593-1612.

Hinnebusch, A.G. (2011) Molecular mechanism of scanning and start codon selection in eukaryotes. Microbiol Mol Biol Rev, 75, 434-467.

Hopper, A. (2013) Transfer RNA post-transcriptional processing, turnover, and subcellular dynamics in the yeast Saccharomyces cerevisiae. Genetics, 194, 4367

Huot, J.L., Enkler, L., Megel, C., Karim, L., Laporte, D., Becker, H.D., Duchêne, A.M., Sissler, M. and Maréchal-Drouard, L. (2014) Idiosyncrasies in decoding mitochondrial genomes. Biochimie, 100C, 95-106.

Jackson, C., Clayden, S. and Reyes-Prieto, A. (2015) The Glaucophyta: the blue-green plants in a nutshell. Acta Societatis Botanicorum Poloniae, 84, 144-165.

Lavrov, D.V. and Pett, W. (2016) Animal mitochondrial DNA as we do not know lt: mtgenome organization and evolution in nonbilaterian lineages. Genome Biol Evol, 8, 2896-2913.

Leblanc, C., Boyen, C., Richard, O., Bonnard, G., Grienenberger, J.M. and Kloareg, B. (1995) Complete sequence of the mitochondrial DNA of the rhodophyte Chondrus crispus (Gigartinales). Gene content and genome organization. $J \mathrm{Mol}$ Biol, 250, 484-495.

Lechner, M., Rossmanith, W., Hartmann, R.K., Tholken, C., Gutmann, B., Giegé, P. and Gobert, A. (2015) Distribution of ribonucleoprotein and protein-only RNase P in eukarya. Mol Biol Evol, 32, 3186-3193.

Mörl, M. and Marchfelder, A. (2001) The final cut. The importance of tRNA 3'processing. EMBO Rep, 2, 17-20. 
Ohyama, K. (1996) Chloroplast and mitochondrial genomes from a liverwort, Marchantia polymorpha - Gene organization and molecular evolution. Bioscience, biotechnology, and biochemistry, 60, 16-24.

Ojala, D., Merkel, C., Gelfand, R. and Attardi, G. (1980) The tRNA genes punctuate the reading of genetic information in human mitochondrial DNA. Cell, 22, 393-403.

Ojala, D., Montoya, J. and Attardi, G. (1981) tRNA punctuation model of RNA processing in human mitochondria. Nature, 290, 470-474.

Pearce, S.F., Rebelo-Guiomar, P., D'Souza, A.R., Powell, C.A., Van Haute, L. and Minczuk, M. (2017) Regulation of Mammalian Mitochondrial Gene Expression: Recent Advances. Trends Biochem Sci, 42, 625-639.

Price, D.C., Chan, C.X., Yoon, H.S., Yang, E.C., Qiu, H., Weber, A.P., Schwacke, R., Gross, J., Blouin, N.A., Lane, C., Reyes-Prieto, A., Durnford, D.G., Neilson, J.A., Lang, B.F., Burger, G., Steiner, J.M., Loffelhardt, W., Meuser, J.E., Posewitz, M.C., Ball, S., Arias, M.C., Henrissat, B., Coutinho, P.M., Rensing, S.A., Symeonidi, A., Doddapaneni, H., Green, B.R., Rajah, V.D., Boore, J. and Bhattacharya, D. (2012) Cyanophora paradoxa genome elucidates origin of photosynthesis in algae and plants. Science, 335, 843-847.

Roger, A.J., Munoz-Gomez, S.A. and Kamikawa, R. (2017) The origin and diversification of mitochondria. Curr Biol, 27, R1177-R1192.

Ryabova, L.A., Pooggin, M.M. and Hohn, T. (2006) Translation reinitiation and leaky scanning in plant viruses. Virus Res, 119, 52-62.

Salinas-Giegé, T., Cavaiuolo, M., Cognat, V., Ubrig, E., Remacle, C., Duchêne, A.M., Vallon, O. and Maréchal-Drouard, L. (2017) Polycytidylation of mitochondrial mRNAs in Chlamydomonas reinhardtii. Nucleic Acids Res, 45, 12963-12973.

Sieber, F., Placido, A., El Farouk-Ameqrane, S., Duchene, A.M. and MarechalDrouard, L. (2011) A protein shuttle system to target RNA into mitochondria. Nucleic Acids Res, 39: e96

Sloan, D.B., Alverson, A.J., Chuckalovcak, J.P., Wu, M., McCauley, D.E., Palmer, J.D. and Taylor, D.R. (2012) Rapid evolution of enormous, multichromosomal genomes in flowering plant mitochondria with exceptionally high mutation rates. PLoS Biol, 10, e1001241. 
Smith, D.R. and Keeling, P.J. (2015) Mitochondrial and plastid genome architecture: Reoccurring themes, but significant differences at the extremes. Proc Natl Acad Sci, USA, 112, 10177-10184.

Torres, A.G., Piñeyro, D., Rodriguez-Escribà, M., Camacho, N., Reina, O., SaintLéger, A., Filonava, L., Battle, E., and Ribas de Pouplana, L. (2015) Inosine modifications in human tRNAs are incorporated at the precursor tRNA level. Nucleic Acids Res, 43, 5145-5157.

Unseld, M., Marienfeld, J.R., Brandt, P. and Brennicke, A. (1997) The mitochondrial genome of Arabidopsis thaliana contains 57 genes in 366,924 nucleotides. Nature Genet, 15, 57-61.

Varre, J.S., D'Agostino, N., Touzet, P., Gallina, S., Tamburino, R., Cantarella, C., Ubrig, E., Cardi, T., Drouard, L., Gualberto, J.M. and Scotti, N. (2019) Complete Sequence, Multichromosomal Architecture and Transcriptome Analysis of the Solanum tuberosum Mitochondrial Genome. Int J Mol Sci, 20.

Wu, Z., Cuthbert, J.M., Taylor, D.R. and Sloan, D.B. (2015) The massive mitochondrial genome of the angiosperm Silene noctiflora is evolving by gain or loss of entire chromosomes. Proc Natl Acad Sci, USA, 112, 10185-10191.

Yot, P., Pinck, M., Haenni, A.L., Duranton, H.M. and Chapeville, F. (1970) Valinespecific tRNA-like structure in turnip yellow mosaic virus RNA. Proc Natl Acad Sci, USA, 67, 1345-1352.

\section{Legend to figures}

Figure 1. Schematic representation of the organization of the C. paradoxa mitochondrial tRNA genes. Red arrows depict tRNA genes represented by the one-letter amino acid code. When isoacceptor tRNA genes exist, they are numbered. Protein-coding genes and rRNA genes are represented by blue and green arrows, respectively. Distances between protein-coding genes or ribosomal RNA genes and the closest tRNA genes are indicated in base pair. 
Figure 2. The 3' extremity of mRNAs lies at the 3'-end of the closest downstream mature tRNA. (A), Sample sequence of one of the clones obtained from the 3' RACE amplification product (Figure S3) using a primer P specific to rps4 mRNA (see Table S1). The 3'- extremity of rps4 CDS and the tRNA ${ }^{\text {Tyr }}$ are indicated by blue and black arrows. The CCA triplet not encoded by the tRNA gene and added at the 3' extremity of the tRNA is indicated by a grey arrow and highlighted in red. (B), Mapped rps4 mRNA termini. Black arrows indicate the 3 '-termini of 47 sequences obtained as described in (A) (Figure S9). The number of clones corresponding to each type of extremity is given. The CCA triplet is in red. (C), Mapped 5S rRNA termini. Black arrows indicate the $3^{\prime}$ termini of 65 sequences obtained as described in (A) with a primer specific to 5S rRNA (Table S1). The number of clones corresponding to each type of extremity is given. (D), Analysis of the 3'- termini of 6 mRNAs. On the left, the 6 mRNAs are represented by colored arrows followed by their downstream tRNA. On the right, the histogram summarizes, for each mRNA, the percentage of 3'- extremities located at different positions: within the mRNA, in the intergenic region, within the tRNA, at the 3'- CCA end of the tRNA. (E), Ethidium bromide $(\mathrm{EtBr})$ stained gel of total $C$. cyanophora RNA fraction separated on denaturing formaldehyde agarose gel and analyzed by northern (No) using a probe specific to nad4. (F), Boxplot representing the lengths of the 5'- UTR of four mRNAs.

Figure 3. The 3 '- termini of cox 1 and nad 7 mRNAs are preferentially located within or at the 3'- end of stem-loop structures. A tRNA gene ( $\mathrm{P}$ and S1, respectively) is present upstream of cox1 and nad7 protein-coding genes but not downstream. The $3^{\prime}$ coding sequences of cox 1 and nad7 CDS are depicted with pale grey arrows and the UAA stop codon in red. Stem-loop structures found downstream the two mRNAs according to RNAfold program are indicated. Positions of the 3'- termini identified by 3' RACE are depicted by black arrow heads and the number of clones for each type of extremity is given. The cox1 3' RACE data were previously published and reanalyzed (Salinas-Giegé et al., 2017).

Figure 4. Existence of long polycistronic mRNAs. (A), Schematic representation of adjacent protein-coding genes found on the $C$. paradoxa mitochondrial genome. The numbers on the top of the genes indicate the distance between protein-coding genes in 
bp. The red numbers indicate the overlapping between two genes. (B), cRT-PCR analyses of the region comprising nad1-rps13-rps11-rpl5-orf99-rpl6-tRNAMete. After circularization of total nucleic acids in the presence of T4 RNA ligase, cDNA was synthesized using either primers $\mathrm{P} 1$ or $\mathrm{P} 3$ and PCR products amplified using pairs of primers $\mathrm{P} 1 / \mathrm{P} 2, \mathrm{P} 3 / \mathrm{P} 4$, or P1/P4 (Table S1) were fractionated on an agarose gel in the presence of ethidium bromide. (C), An illustration of one of the clones from the cRT-PCR product obtained with P1/P4 is shown. tRNAMete is depicted by a black arrow. The 3'- and 5'- extremities of rpl6 and nad1 respectively are in blue and their corresponding 3'- and 5'- UTR in grey. The post-transcriptionally added CCA triplet is in red. (D), Screenshot of the visualization of small RNA-seq data retrieved from SRA (SRR363339) on the nad1rps13-rps11-rpl5-orf99-rpl6 region of $C$. paradoxa mitochondrial genome, using Geneious genome browser.

Figure 5. Precursor tRNAs are correctly processed into mature tRNAs. (A), Scheme of the cRT-PCR analysis. Total nucleic acids were analyzed using primers P1 and P2. After circularization in the presence of T4 RNA ligase, CDNA was synthesized in the presence of $P 1$, and PCR products were amplified using $P 1$ and $P 2$. The sequences of $P 1$ and $P 2$ are presented in Table S1. (B), Ethidium bromide-stained gel of the PCR products amplified using pairs of primers P1/P2 specific to tRNA ${ }^{A s n}(N)$, tRNA ${ }^{\text {Tyr }}(Y)$, tRNA ${ }^{\text {Leu3 }}$ (L3) and tRNA ${ }^{G l y}(G)$. PCR products corresponding to monomer are marked with a red star. Note that due to the efficiency of the cDNA synthesis step, dimers to multimers are also formed, as visualized by ladders generated and as confirmed by sequencing. Lane $M$ shows the migration of the DNA ladder. An illustration of one clone from the CRT-PCR product obtained for tRNALeu3 (L3) is shown. The 3'- and 5'- extremities of the tRNA molecule are presented and the CCA triplet is framed. Mature tRNAs were also identified for tRNA ${ }^{A s n}$, tRNA $A^{T y r}$ and tRNA $A^{\text {Gly }}$. Complete sequence samples are presented as supplemental information (Figure S4). (C), Ethidium bromide ( $E \mathrm{tBr}$ ) stained gel of total $C$. cyanophora RNA fraction separated on denaturing formaldehyde agarose gel and analyzed by northern (No) using probes specific to tRNA ${ }^{\text {Leu3 }}$ or tRNA ${ }^{\text {Asn. }}$. Signals corresponding to tRNA $A^{\text {Leu3 }}$ or tRNA ${ }^{A s n}$ are indicated and no signals corresponding to nad4- tRNALeu3 (circa $1580 \mathrm{nt}$ ) or nad5- tRNAAsn (circa $2100 \mathrm{nt}$ ) are visible. Note that the blot used with nad4 probe (Figure $2 \mathrm{E}$ ) is the same blot used with tRNALeu3 probe. 
Figure 6. Model of C. paradoxa mitochondrial mRNA and tRNA processing. (A), Biogenesis of mature tRNAs: primary transcripts undergo 5'- and 3 '- end processing by RNase $\mathrm{P}$ first and then RNase Z; it is followed by the addition of the CCA by a tRNAnucleotidyltransferase (CCase). This process represents the main pathway. Other posttranscriptional tRNA modifications, not described here, must also occur. Such mature tRNAs are functional for translation. In parallel, the 3'- ends of mRNAs are accessible to exonucleases and degraded. (B), Stabilization of mature mRNA: in a minority of cases, the primary transcript is first cleaved by RNase $Z$, and the CCA triplet is added. This likely strongly inhibits RNase $\mathrm{P}$ activity, thus allowing the presence of a strong folded structure stabilizing the $3^{\prime}$-end of mRNAs by impeding the action of exonucleases. We can hypothesize that such stable mRNAs are engaged in translation. 

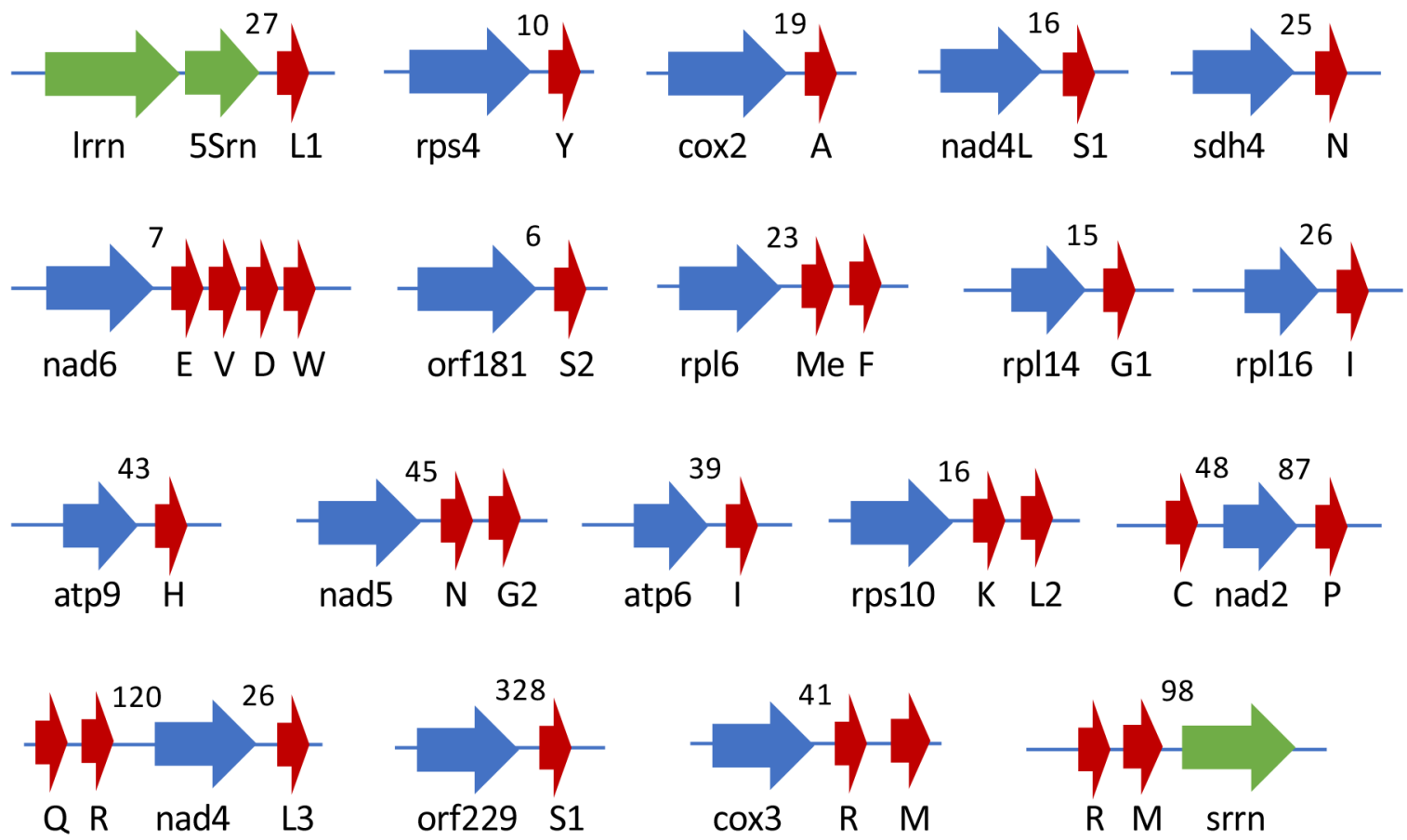

tpj_15222_f1.tif

This article is protected by copyright. All rights reserved 
A

$\stackrel{\mathrm{P}}{\rightarrow} \quad \mathrm{rps} 4 \quad$ tRNA Tyr

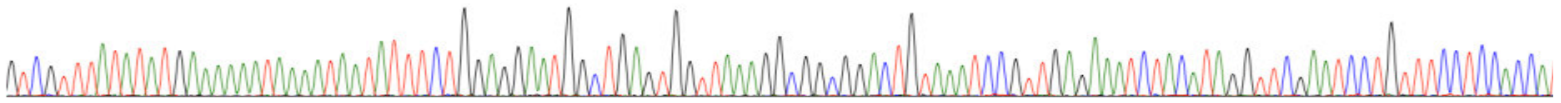

B

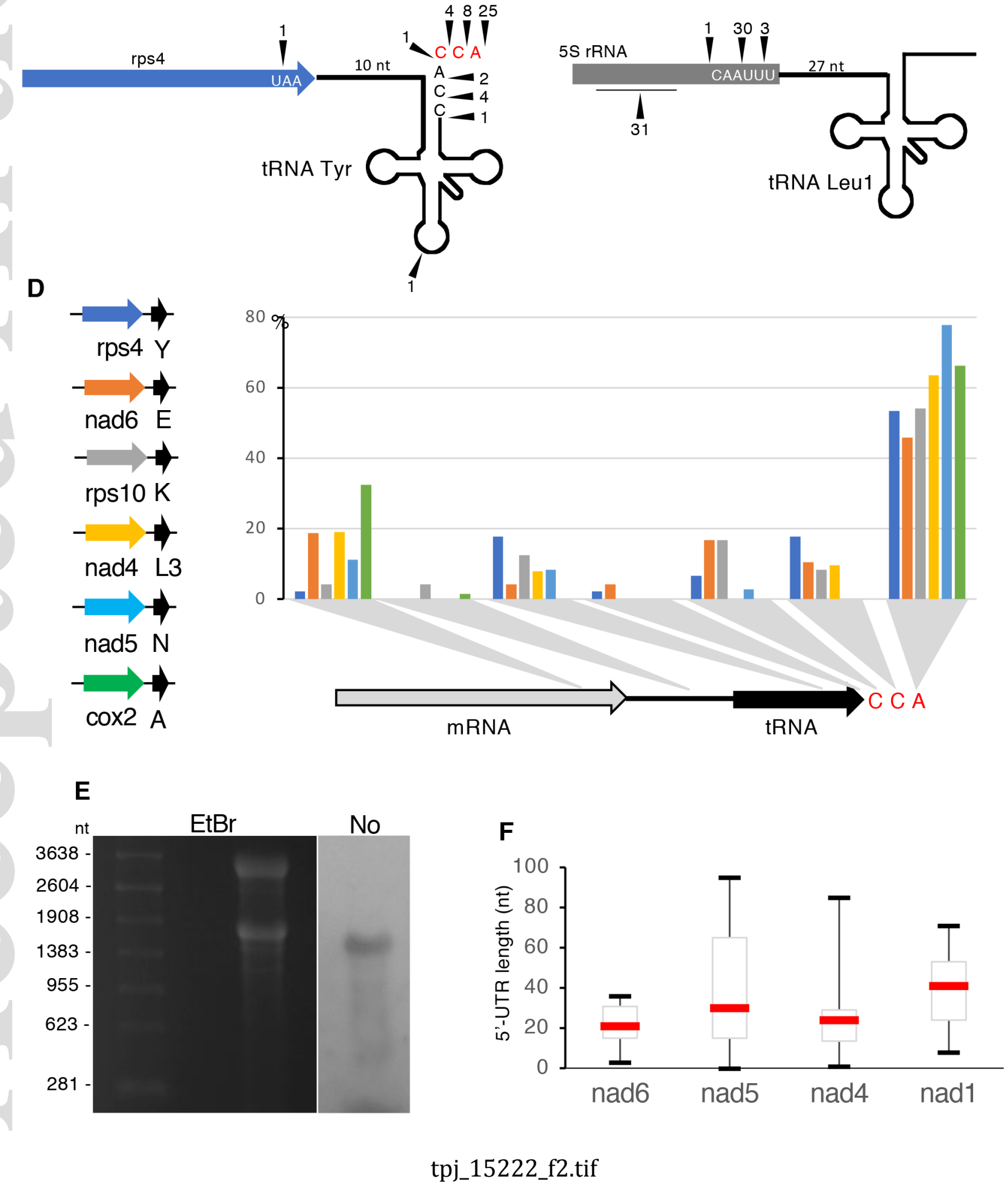

D
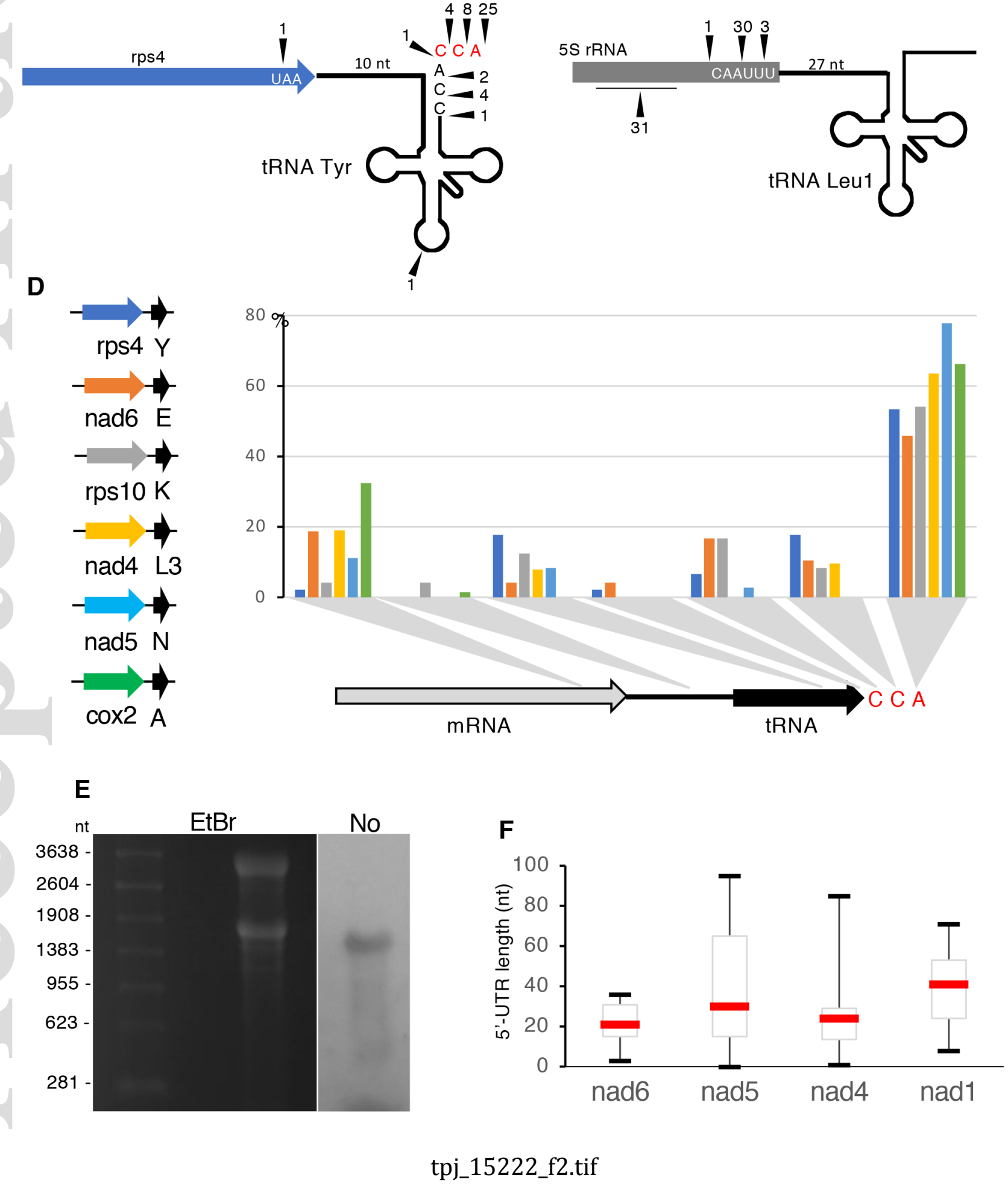

C

This article is protected by copyright. All rights reserved 


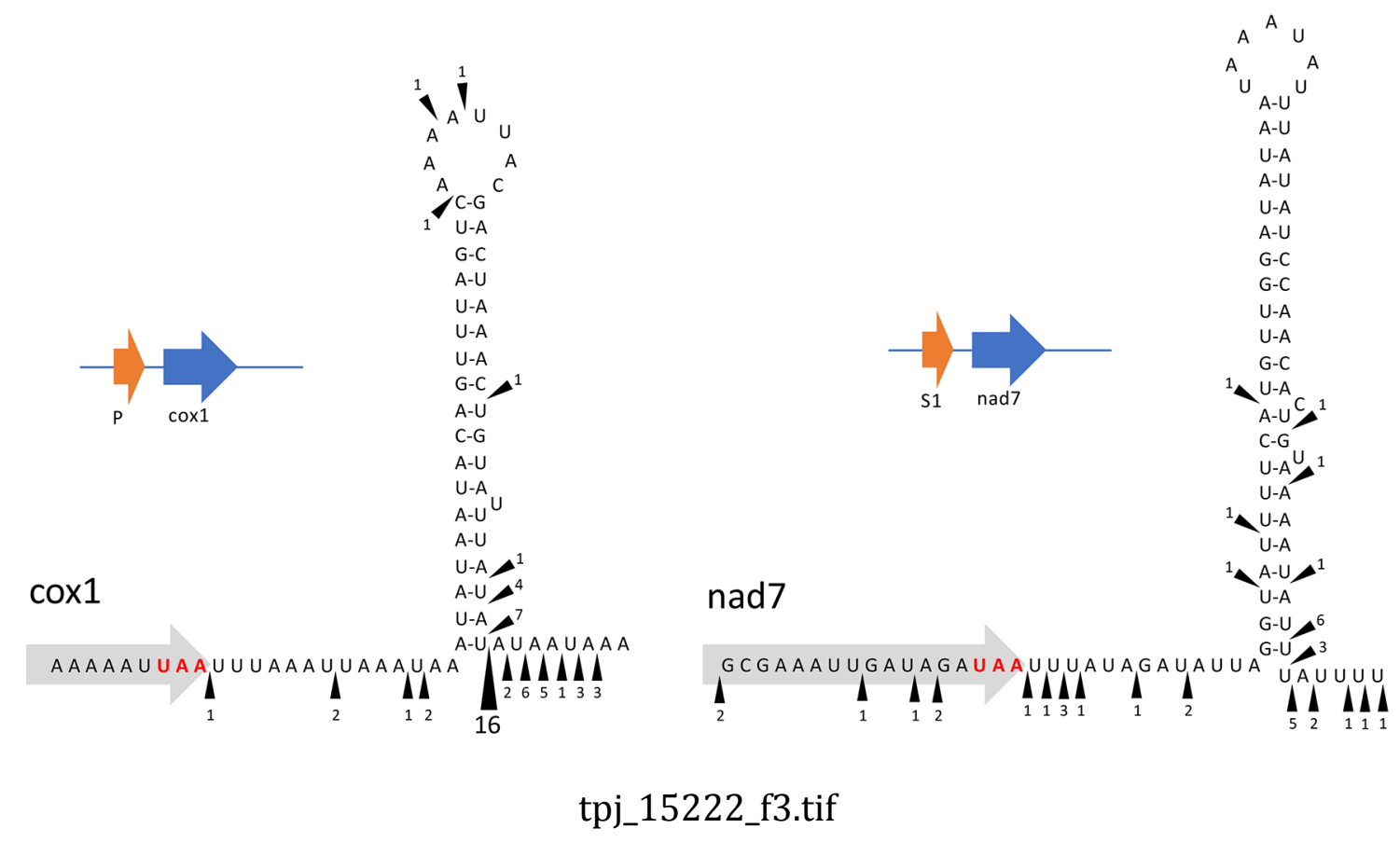

This article is protected by copyright. All rights reserved 
A
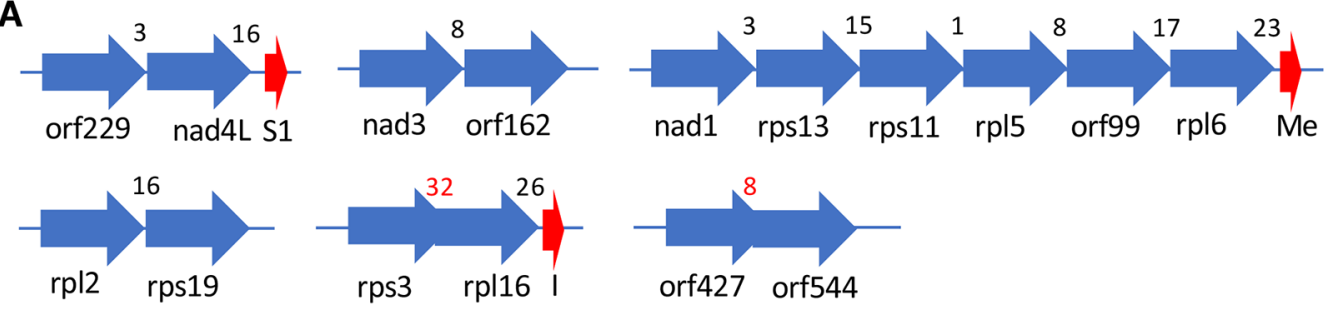

B
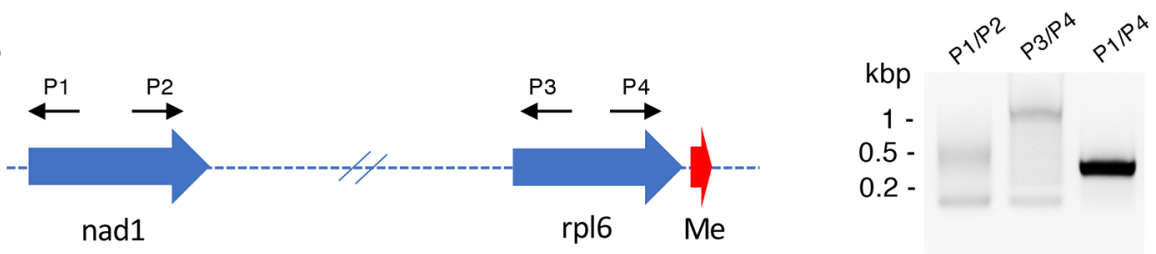

C

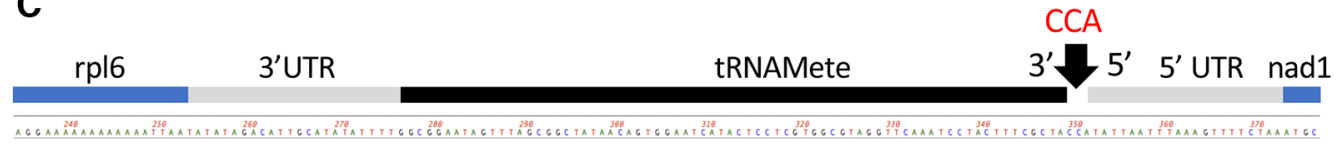

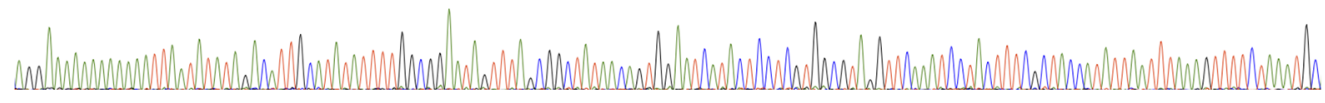

D



tpj_15222_f4.tif 
A

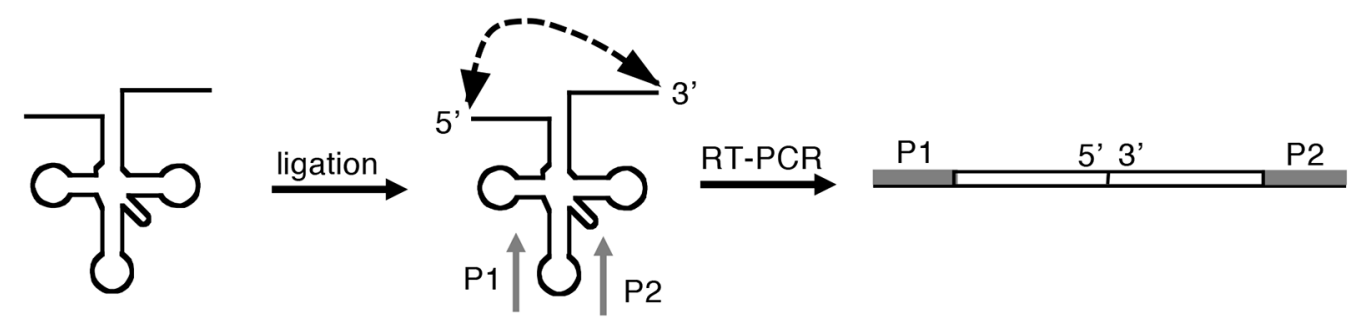

B
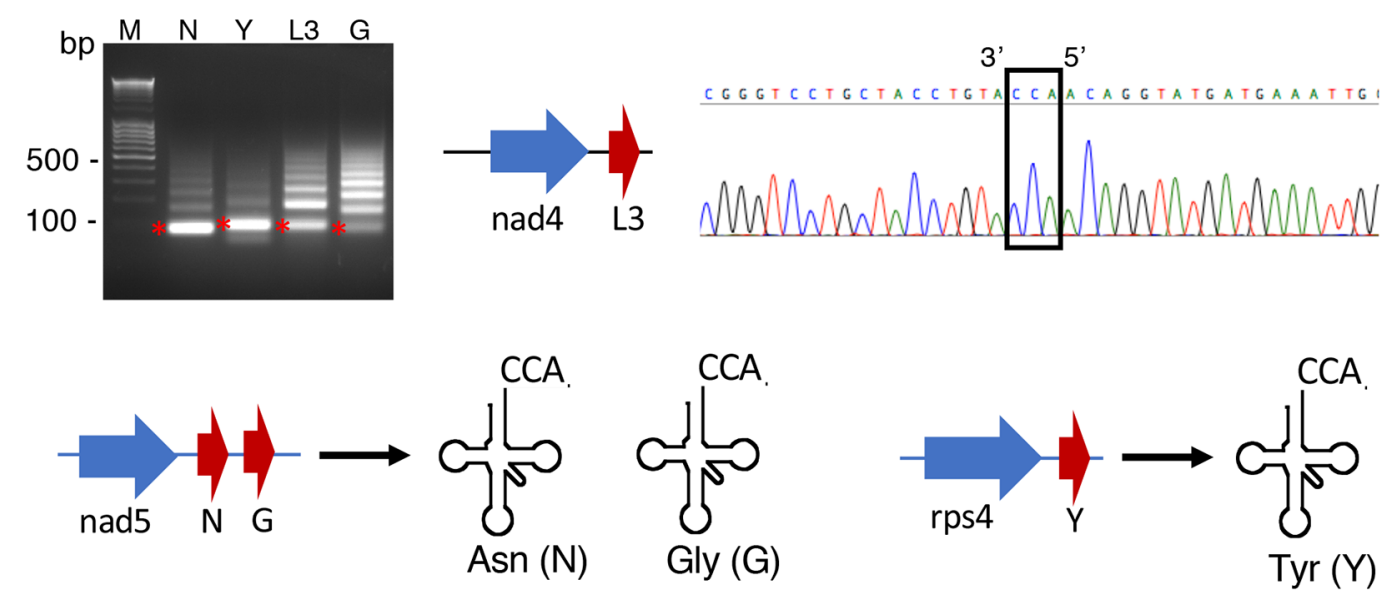

C
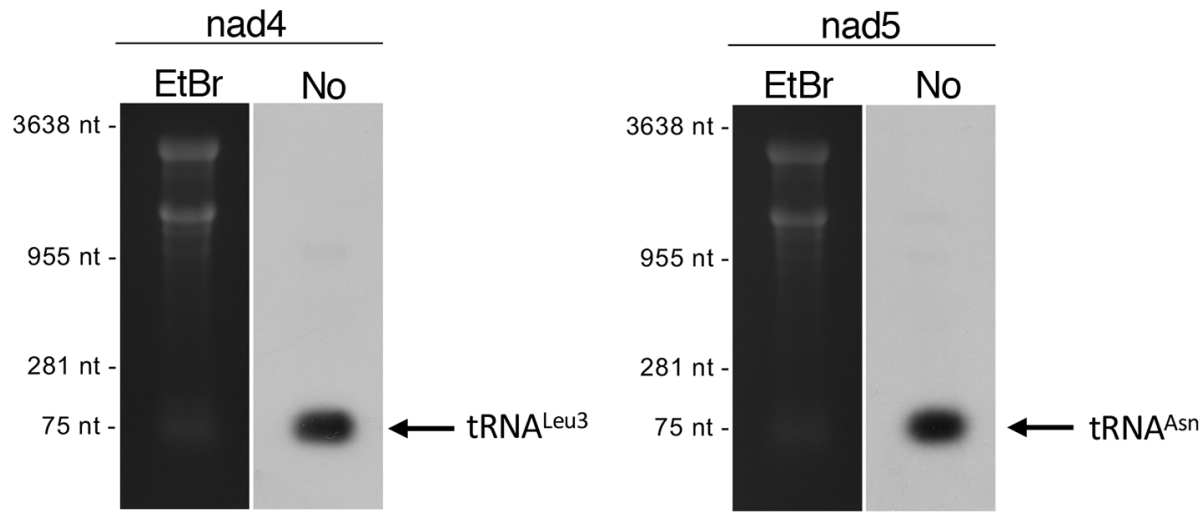

tpj_15222_f5.tif

This article is protected by copyright. All rights reserved 


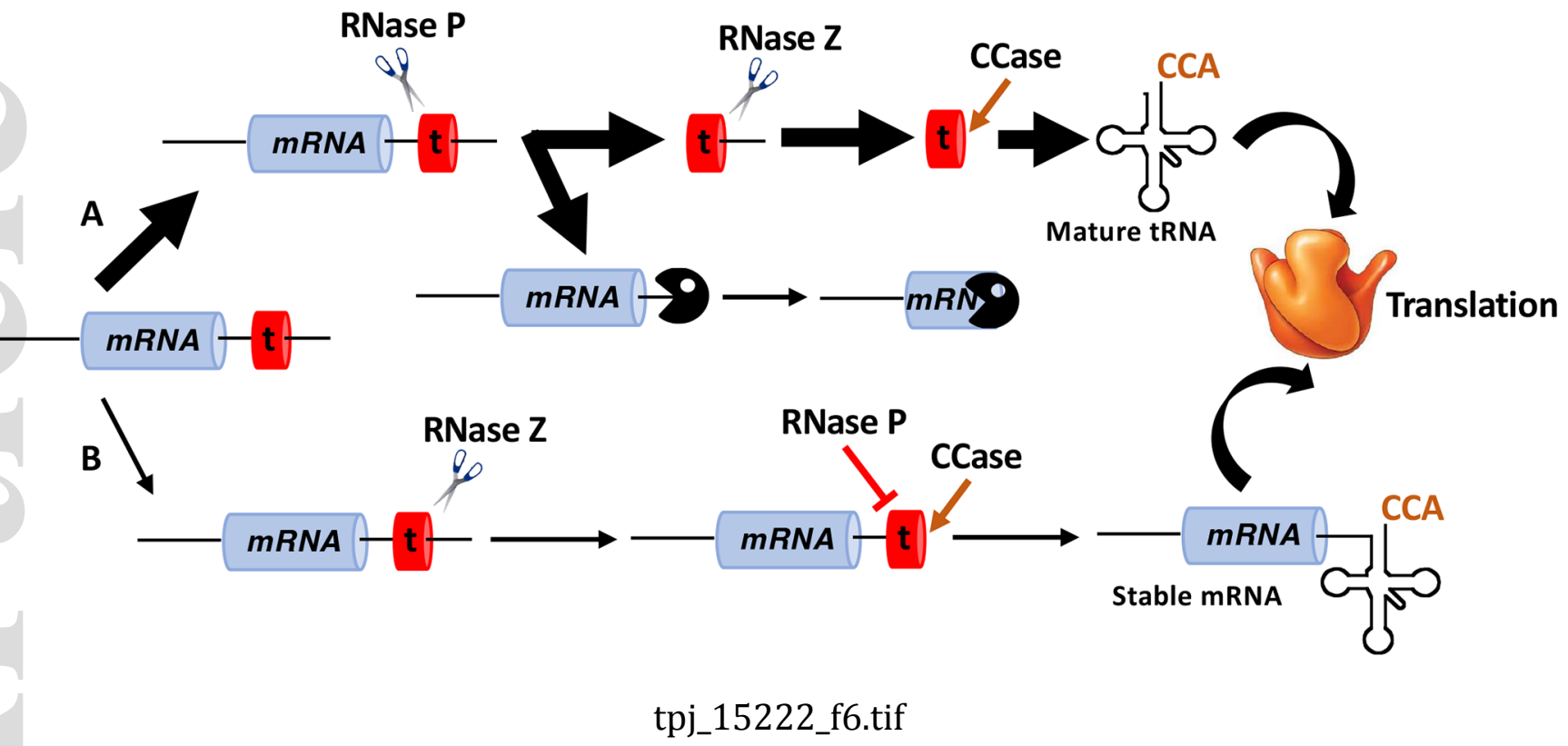

This article is protected by copyright. All rights reserved 